\title{
Oral epithelial dysplasia and oral cancer prevalence in routine white lesion biopsies - a 6-year retrospective study
}

\author{
Maciej Kuzio ${ }^{1, A-D \oplus}$, Karolina Wapniarska ${ }^{1, A-B, D \oplus}{ }^{\text {, Marian Danilewicz }}{ }^{1, E-F} \oplus$, Natalia Lewkowicz ${ }^{1, A, D-F} \oplus$ \\ ${ }^{1}$ Medical University, Łódź, Poland \\ A - Research concept and design, B - Collection and/or assembly of data, C - Data analysis and interpretation, \\ $D$ - Writing the article, E-Critical revision of the article, F- Final approval of article
}

Kuzio M, Wapniarska K, Danilewicz M, Lewkowicz N. Oral epithelial dysplasia and oral cancer prevalence in routine white lesion biopsies a 6-year retrospective study. J Pre Clin Clin Res. 2020; 14(3): 63-68. doi: 10.26444/jpccr/125393

\begin{abstract}
Introduction. Leukoplakia and oral lichen planus (OLP) are common diseases manifesting as white lesions that are considered potentially malignant disorders (PMD). Epithelial dysplasia may be an early sign of potency for the future transformation into oral squamous cell carcinoma (OSCC). A routine biopsy and close observation are recommended for persistent white oral lesions. As frictional keratosis may mimic oral leukoplakia, the question arises:Is there a need for a biopsy of persistent white lesion of traumatic origin?

Materials and methods. Data from 643 oral tissue biopsies were retrospectively analyzed. A total of $176(27.37 \%)$ results with provisional diagnosis of leukoplakia (36 cases), OLP (77 cases) and frictional keratosis ( 63 cases) were selected. Retrospective data collected included age, gender, smoking status, provisional and histopathological diagnosis. The data was analyzed to assess the prevalence of epithelial dysplasia and OSCC in terms of age, gender and smoking status.

Results. Five (2.84\%) cases of OSSC were reported, all of them were graded as G1; four cases of OSCC were found in clinically defined leukoplakia lesions; one case of OSCC (1.3\%) was found in OLP biopsy; epithelial dysplasia was reported in 5 lesions (2.84\%) provisionally diagnosed as OLP (3 cases), and leukoplakia in 2 cases. No dysplasia or OSCC were found in the lesions diagnosed as frictional keratosis.

Conclusions. Epithelial dysplasia and OSCC may be found in leukoplakia or OLP lesions not initially suspected of any malignancy. In some cases, clinical features are not sufficient to diagnose a lesion without histopathology. Frictional keratosis is easily identified by clinicians, and may not require a biopsy in every case. Clinical and histopathological evaluation of the white lesions still needs improvement.
\end{abstract}

\section{Key words}

oral lichen planus, oral cancer, leukoplakia, oral epithelial dysplasia, frictional keratosis

\section{INTRODUCTION}

Oral cancer is considered to be the sixth most prevalent type of cancer worldwide. Approximately $90 \%$ of oral malignant neoplasms are squamous cell carcinomas (OSCC) [1]. Although OSCC may develop de novo, some of them originate from potentially malignant disorders (PMD) which commonly appear in the oral cavity as white lesions, such as oral leukoplakia and oral lichen planus (OLP). These conditions may cause diagnostic problems for clinicians, usually being miscategorized as other benign lesions, especially frictional keratosis [2]. It is advisable to perform a biopsy and confirm a provisional diagnosis for any persistent white lesion, especially if its clinical appearance is not specific [3].

Currently, one of the main predictive tests of malignant transformation is the presence of epithelial dysplasia and its severity. This factor, however, is not fully decisive, as dysplasia may regress and its presence is not mandatory for malignant transformation in the future. [4]. Some cases of non-dysplastic lesions may develop OSCC omitting the stage of dysplasia [5]. Dysplasia can also be detected in other inflammatory conditions not related to PMDs, such as candidiasis [6].

Address for correspondence: Maciej Kuzio, Medical University of Łódź, Poland E-mail: maciej.kuzio@stud.umed.lodz.pl

Received: 15.05.2020; accepted: 14.07.2020; first published: 10.08.2020
The classification of oral leukoplakia and leukoplakia-like lesions has been the subject of discussion in the literature for many years. Both clinical and histopathological diagnostic aspects may cause confusion in establishing the final diagnosis. van der Waal has recently proposed using a combination of the 1978 and the 2005 WHO definitions of oral leukoplakia as following: 'A predominantly white patch or plaque that cannot be characterized clinically or pathologically as any other disorder; oral leukoplakia carries an increased risk of cancer development either in or close to the area of leukoplakia or elsewhere in the oral cavity or the head-and-neck region' [7].

Leukoplakia is a clinical diagnosis that can only be made by exclusion; thus, this definition is misleading and confusing. In many cases, leukoplakia is associated with tobacco use, but idiopathic cases have also been frequently reported [8].

Lichen planus is a chronic mucocutaneous disease. It may affect oral mucosa with a high tendency for bilateral appearance on buccal mucosa $[9,10]$. Reticular OLP is the most common type, with a network of fine white lines; however, the clinical picture may be mixed involving papular, plaque-like, erosive or atrophic areas [11]. Clinical diagnosis should be usually supported by a biopsy and histopathological examination.

Frictional keratosis is a benign lesion caused by the chronic rubbing of two surfaces against each another, which increases keratinization and results in the clinical appearance of a 
white patch [12]. The most common sites of appearance are alveolar ridges, buccal and labial mucosa, but the tongue can also be affected. The prevalence of frictional keratosis is difficult to assess as it can be easily mistaken with leukoplakia. Some reports suggest that it may appear in about $5 \%$ of the population [2]. After elimination of the possible mechanical irritation, the lesion should disappear within a period of 4-8 weeks. There do not appear to be any reports of malignant transformation in frictional keratosis.

Clinically, leukoplakia and lichen planus, especially the plaque type, could mimic each other and cause diagnostic difficulties. Furthermore, a similar clinical appearance can be seen in cases of frictional keratosis [13]. As both leukoplakia and OLP are PMDs, clear criteria are needed to identify those lesions that might progress to OSCC. This study aims to assess the rate of epithelial dysplasia and oral squamous cell carcinoma found in routine histopathology of white lesions.

\section{MATERIALS AND METHODS}

The records of patients who had been referred for diagnosis and treatment of oral pathologies in the period January 2013 - December 2019 were examined, from which cases with a provisional diagnosis of leukoplakia, OLP or frictional keratosis were selected. Leukoplakia was diagnosed by finding a symptomless white lesion, mainly in a smoking patient, the presence of which could not be explained by other means [7]. Oral lichen planus was diagnosed by finding papules that formed a network of white lines along which may also occur red areas of atrophy or erosions [11]. Frictional keratosis was diagnosed as white patches in areas prone to irritation, such as alveolar ridges, cheeks or lips, where an irritating factor was strongly suspected [12]. Retrospective data collected included age, gender, smoking status, provisional and histopathological diagnosis. Biopsy results that were not definitive and patients' records with missing data were excluded from the study. Patients with a strong initial suspicion of OSCC were also excluded, and were referred to the Department of Oral and Maxillofacial Surgery to ensure a quick diagnostic process.

The data was analyzed using descriptive statistics to assess the prevalence of epithelial dysplasia and OSCC in terms of age, gender, and smoking status, by means of Statistica software.

\section{RESULTS}

Clinical and histopathological data related to 643 consecutive biopsies from the oral cavity were retrospectively analyzed. A total of $176(27.37 \%)$ biopsy results with clinically determined white lesions that constituted 36 leukoplakia cases, 77 OLP cases and 63 frictional keratosis cases were selected. The study group included 125 (71.02\%) females and 51 (28.98\%) males. Mean age at presentation was $61.94 \pm 14.84$ years for women and $51.76 \pm 10.70$ years for men. The youngest patient was aged 18 years, and the oldest was 87 at the time of biopsy. Smoking habit was reported in 76 patients (43.18\%).

Table 1 shows the histopathology of all cases. Among all histopathological diagnoses, 5 (2.84\%) cases of OSSC were reported, all of them were graded as G1. OSCC was found on the lips ( 2 cases -1 upper, 1 lower), floor of the mouth (2
Table 1. Histopathological diagnoses of all 176 white lesion biopsies

\begin{tabular}{lcc}
\hline Histopathological diagnosis & Total No. & Percentage of cases \\
\hline OLP & 76 & $43.18 \%$ \\
\hline Epithelial hyperplasia & 6 & $3.41 \%$ \\
\hline Epithelial dysplasia & 5 & $2.84 \%$ \\
\hline OSCC & 5 & $2.84 \%$ \\
\hline Frictional keratosis & 84 & $47.73 \%$ \\
\hline
\end{tabular}

cases) and hard palate (1 case). Four of five cases of OSCC were found in clinically defined leukoplakia lesions; thus, the prevalence of OSCC in leukoplakia was $11.11 \%$. One case of OSCC (1.3\%) was found in OLP biopsy. Three of four cases of OSCC in leukoplakia lesions were smokers, and the patient with a provisional diagnosis of OLP was a nonsmoker. Clinical and histopathological diagnoses overlap and its relation to the cases of dysplasia/OSCC are presented in the Table 2.

Table 2. Distribution of white lesions, histopathological agreement, dysplasia, OSCC and smoking status $(n=176)$

\begin{tabular}{lccccc}
\hline Provisional diagnosis & Total No. & $\begin{array}{c}\mathrm{H} / \mathrm{P} \\
\text { diagnosis } \\
\text { confirmation }\end{array}$ & $\begin{array}{c}\text { Cases } \\
\text { with } \\
\text { dysplasia }\end{array}$ & $\begin{array}{c}\text { Cases of } \\
\text { OSCC }\end{array}$ & Smokers \\
\hline Leukoplakia & 36 & $8(22.22 \%)$ & $2(5.56 \%)$ & $\begin{array}{c}4 \\
(11.11 \%)\end{array}$ & $\begin{array}{c}32 \\
(88.89 \%)\end{array}$ \\
\hline OLP & 77 & $64(83.12 \%)$ & $3(3.9 \%)$ & $1(1.3 \%)$ & $\begin{array}{c}19 \\
(24.68 \%)\end{array}$ \\
\hline Frictional keratosis & 63 & $59(93.65 \%)$ & 0 & 0 & $\begin{array}{c}25 \\
(39.68 \%)\end{array}$ \\
\hline
\end{tabular}

Epithelial dysplasia was reported in 5 white lesions (2.84\%), three of which were provisionally diagnosed as plaque type OLP (Tab. 3). However, the histopathological picture was lacking typical OLP features, and was subsequently considered as epithelial hyperplasia in leukoplakia. The other 2 cases were provisionally diagnosed as leukoplakia, which was not denied by histopathology (Tab. 4, Fig. 1). Four of five patients with dysplasia were smokers. Dysplastic lesions were located on the cheek (4 cases) and gingiva (1 case).

Table 3. Histopathological diagnoses of 77 biopsies provisionally diagnosed as OLP

\begin{tabular}{lcc}
\hline Histopathological diagnosis & Total No. & Percentage of cases \\
\hline OLP & 64 & $83.17 \%$ \\
\hline Epithelial dysplasia & 3 & $3.9 \%$ \\
\hline OSCC & 1 & $1.3 \%$ \\
\hline Frictional keratosis & 9 & $11.69 \%$ \\
\hline
\end{tabular}

Table 4. Histopathological results of 36 biopsies provisionally diagnosed as leukoplakia

\begin{tabular}{lcc}
\hline Histopathological diagnosis & Total No. & Percentage of cases \\
\hline OLP & 8 & $22.22 \%$ \\
\hline Epithelial hyperplasia & 6 & $16.67 \%$ \\
\hline Epithelial dysplasia & 2 & $5.56 \%$ \\
\hline OSCC & 4 & $11.11 \%$ \\
\hline Frictional keratosis & 16 & $44.44 \%$ \\
\hline
\end{tabular}



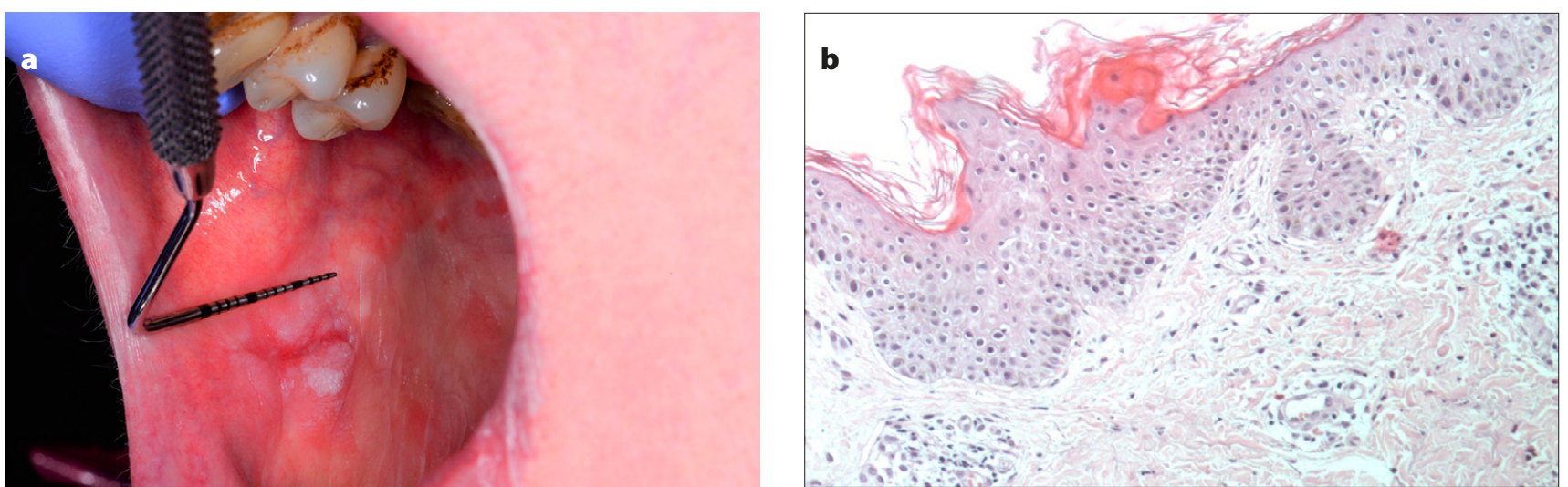

Figure 1. Non-homogeneous leukoplakia on the buccal mucosa. (a) clinical picture, (b) histopathological picture - leukoplakia with dysplasia. Prominent anisonucleosis and hyperchromatosis is visible $(\mathrm{H}+\mathrm{E} 100 \mathrm{x})$

No case of OSCC or epithelial dysplasia was found in the biopsy of persistent frictional keratosis (Tab. 5).

Table 5. Histopathological diagnoses of 63 biopsies provisionally diagnosed as frictional keratosis

\begin{tabular}{lcc}
\hline Histopathological diagnosis & Total No. & Percentage of cases \\
\hline OLP & 4 & $6.35 \%$ \\
\hline Frictional keratosis & 59 & $93.65 \%$
\end{tabular}

\section{DISCUSSION}

The study focused on determining OSCC and epithelial dysplasia rates in oral white lesions with no initial suspicion of malignancy. The lesions were subjected to routine biopsies to confirm or deny provisional diagnosis. When a potential causative factor was detected (smoking, trauma, candida infection), the lesion was observed for the 2-4 weeks needed to obtain a marked improvement or resolution. Cases of strong suspicion of OSCC were referred directly to the Department of Oral and Maxillofacial Surgery for further diagnostics and treatment.

The most common OSCC location was the tongue, followed by the lip and floor of the mouth [14]. In this study, a similar frequency was reported for lip and floor of the mouth, while no case of tongue OSCC originating from a white lesion was reported. A recent study reported that different clinical types of leukoplakia were clinically misdiagnosed in 16,52\% of cases, while malignancies were detected in $6,96 \%$ of cases when it was not initially suspected [15]. In the current study, the frequency of OSCC in leukoplakia was found to be even greater $(11.11 \%)$, while the frequency of OSCC in the total number of white lesions subjected to histopathology was $2.84 \%$. However, this frequency is comparatively low when compared to other study in which the dysplasia rate was $10.4 \%$, and OSCC was found in $14,3 \%$ of all white lesions removed [16].

OLP is a PMD with approximately $1.2-4.9 \%$ rate of transformation. The known risk factors are smoking, alcohol consumption, HCV infection and male gender [17]. In the current study, one case of OSCC (1.3\%) was found in OLP biopsy. This is in accordance with a recent metaanalysis reporting that malignant transformation of OLP was determined in $1.14 \%$ of cases [18].

Since OSCC may originate from PMD, such as leukoplakia or OLP, these lesions are routinely subjected to biopsy and histopathology. Some cases of leukoplakia and plaque type OLP are often confusing to clinicians. For leukoplakia, there are no specific clinical features to differentiate from other white lesions, and histopathological diagnosis is not always decisive. Banóczy et al. attempted to establish the typical histological changes present in leukoplakia lesions. These include abnormalities in keratinization (para-, hyper-, dyskeratosis) (Fig. 2), although neither are indices of premalignancy, changes in epithelial thickness (atrophy) and inappropriate epithelial maturation (dysplasia). In connective tissue, inflammation and hyaline and elacine degeneration were most commonly found [19]. However, some pathologists might deny a diagnosis of leukoplakia in the absence of epithelial dysplasia, which is in disagreement with some recommendations from the dental literature [7]. Moreover, the histopathological picture sometimes cannot be decisive, partially because dysplastic changes are not always visible in the specimen obtained as an incisional biopsy from a large leukoplakic lesion.

The diagnosis a plaque type OLP should be based on the biopsy and histopathology, as microscopic features are usually specific (Fig. 3). The histopathological aspect of lichen planus is described as follows: 'In the area of clinically visible papules, the epithelium is thickened, with acanthosis and hyperkeratosis and liquefactive degeneration of the basal layer. Round or ovoid 'colloid bodies' appear mainly in the spinous layer of epithelium and in the lamina propria. Also a well-defined inflammatory zone in the connective tissue is present' [3]. However, some samples may not show typical features and final diagnosis should be made by evaluating both clinical and histopathological aspects [9].

The presence of epithelial dysplasia is commonly used to evaluate the malignant potential of PMDs. It has been suggested that DNA ploidy analysis or p53 expression may be a more precise method of predicting malignancies, as cases of DNA aneuploidy and mutations in p53 show a higher risk of malignant transformation $[4,20]$. Moreover, Nagler et al. found that biomarker levels in saliva, such as CA125, tissue polipeptyde antigen and Cyfra 21-1, were significantly elevated in oral cancer patients compared to healthy controls [21].

Although oral leukoplakia and frictional keratosis often show similar clinical features, frictional keratosis is not believed to be a PMD. Histological features include hyperparakeratosis and peeling surface with fissures and clefts (Fig. 4). In most cases, lesions are colonized with 

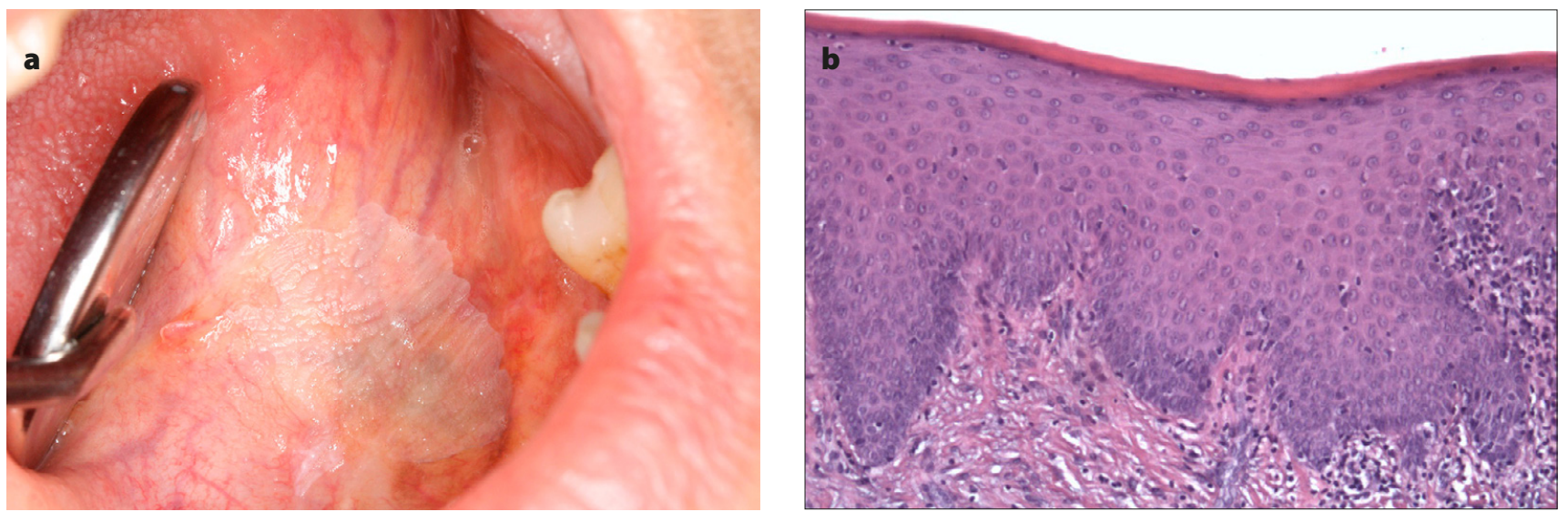

Figure 2. Homogeneous leukoplakia on the floor of the mouth. (a) clinical picture, (b) histopathological picture - no features of epithelial dysplasia (H+E 100x)
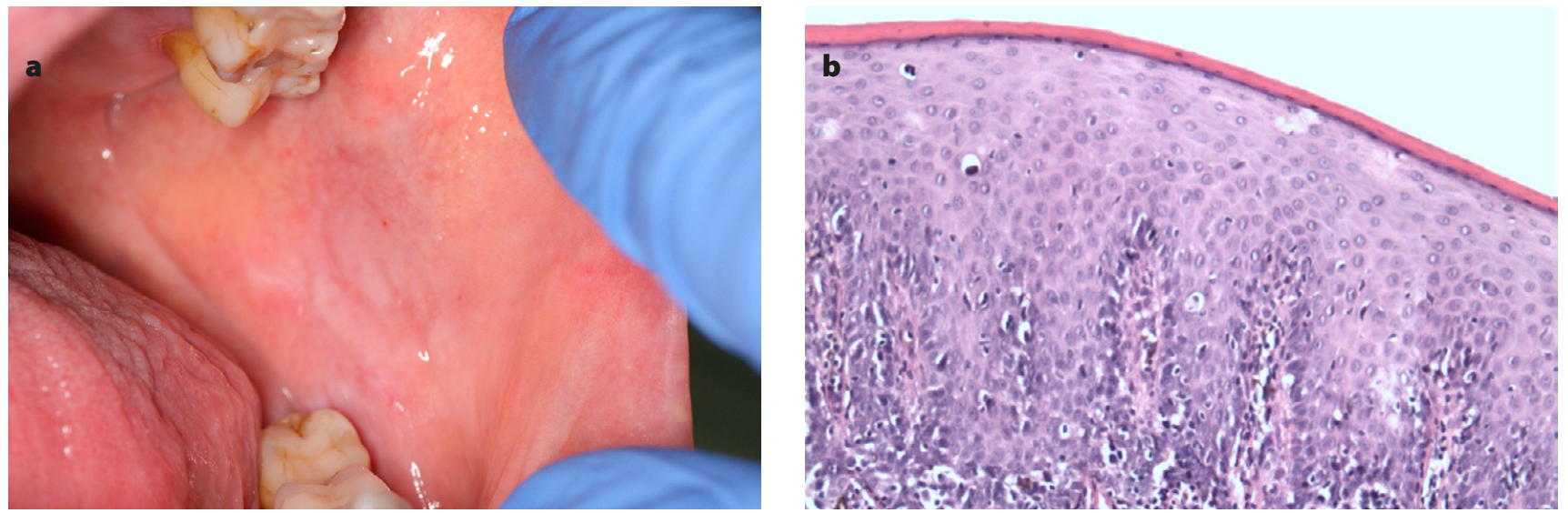

Figure 3. A white diffused lesion on the buccal mucosa - leukoplakia or plaque type OLP. (a) clinical picture, (b) histopathological picture-lichen planus. The epithelium is thin. Note inflammatory infiltrates in subepithelial area $(\mathrm{H}+\mathrm{E} 100 \mathrm{x})$
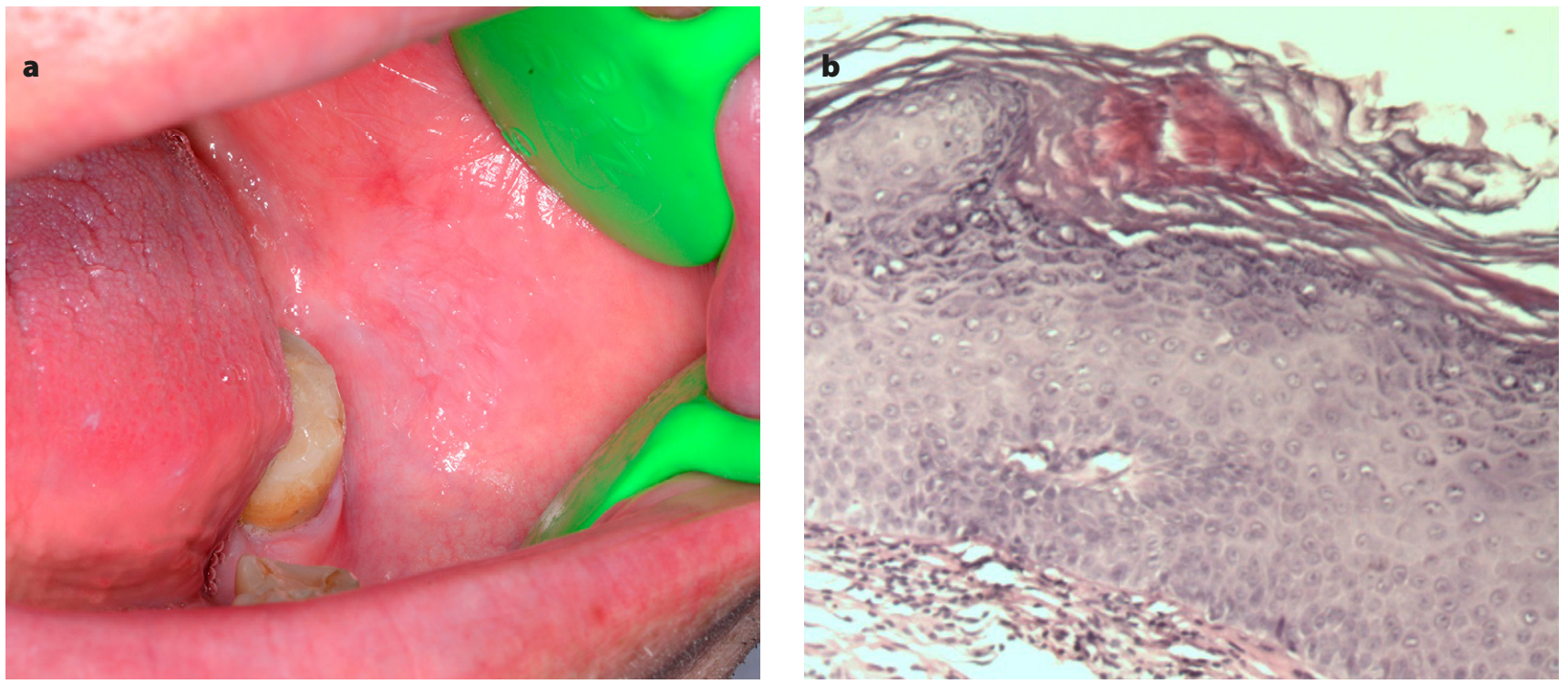

Figure 4. Frictional keratosis on buccal mucosa caused by the sharp edge of a tooth. (a) clinical picture, (b) histopathological picture - no features of epithelial dysplasia $(\mathrm{H}+\mathrm{E}$ 100x)

bacteria and show no signs of inflammation or epithelial dysplasia [22]. In the current study, no case of OSCC or epithelial dysplasia was found in the biopsy of persistent frictional keratosis, confirming that trauma-induced white lesions have no potential for malignant transformation. The biopsies were taken only when the lesion was not markedly improved or completely healed within the period of $2-4$ weeks following elimination of the causative factor. This finding raises the question of unnecessary biopsies in such cases, and clearly demonstrates the need for determining a non-invasive method for white lesion diagnostics. However, Chi et al. reported that some of the biopsied samples of alveolar ridge keratosis (ARK) showed dysplasia [23]. Dysplasia was found only in the patients with confirmed tobacco smoking 
and alcohol consumption; therefore, it was suggested that leukoplakia can mimic ARK, and biopsy of any persistent white lesions is recommended, especially, when other risk factors are present [23].

White lesions are commonly found in the oral cavity and can cause diagnostic difficulties. According to epidemiological studies performed on 17000 Americans, white lesions were found in $27.9 \%$ of individuals [24]. Oral lesions, in spite of similar appearance, may be the result of various diseases, ranging from mechanical trauma, through precancerous lesions to squamous cell carcinoma [25]. Despite having typical and benign clinical features, a biopsy is advised of an oral white lesion when a patient presents himself for the first time. Familial history, trauma, drug intake, habits and patient's complaints should all be included in the provisional diagnosis process $[26,27]$. If the lesion is asymptomatic, clinician is often the first person to diagnose the disease. If the symptoms are present, it is important to collect relevant information about the condition: the date of first appearance, localization, duration, previous treatment and patient's habits [26]. White lesions for which a possible causative factor has been identified, e.g. smokers' lesion or frictional keratosis could be observed for 4-8 weeks after removal of the suggested cause to achieve spontaneous resolution. However, even such a period is already a long one in the case of a squamous cell carcinoma, a carcinoma in situ or severe epithelial dysplasia. Therefore, in the case of non-homogenous, symptomatic lesions, a biopsy is strongly recommended before elimination of possible causative factors and observation [7].

\section{CONCLUSIONS}

Clinical evaluation of white lesions still needs improvement and the histopathological criteria of the diagnosis require further standardization, related not only to dysplasia. In some lesions, oral cancer develops even without dysplasia in earlier biopsies; for this reason, a regular clinical and histopathological evaluation allows finding a potential malignancy at an early stage [28]. Further research of samples that showed features of malignancy might help in developing new diagnostic methods that should be precise and rapid. Molecular biomarkers may be valuable in the evaluation of leukoplakia for both diagnostic and prognostic aspects. So far, no method employing biological markers is available, but in future, the monitoring of many genes simultaneously might allow the prediction of the risk of malignant transformation of precancerous lesions. These methods might be more reliable than those depending on the monitoring of epithelial dysplasia [28]. A non-invasive tool that would help to reduce the number of unnecessary biopsies would greatly improve the cost effectiveness, as well as patient compliance with screening programmes for high risk patients. Moreover, such a tool might enable cost effective screening of the general population. So far, every case of atypical white lesions of unknown origin or those that do not respond to treatment, should be biopsied to ensure early detection of potential malignancy.

\section{REFERENCES}

1. Warnakulasuriya S. Global epidemiology of oral and oropharyngeal cancer. Oral Oncol. 2009; 45: 309-316. https://doi.org/10.1016/j. oraloncology.2008.06.002

2. Müller S. Frictional Keratosis, Contact Keratosis and Smokeless Tobacco Keratosis: Features of Reactive White Lesions of the Oral Mucosa. Head Neck Pathol. 2019; 13(1): 16-24. https://doi.org/10.1007/s12105018-0986-3

3. Bánóczy J. Oral leukoplakia and other white lesions of the oral mucosa related to dermatological disorders. J Cutan Pathol. 1983; 10(4): 238-56. https://doi.org/10.1111/j.1600-0560.1983.tb01490.x

4.Zaini ZM, McParland H, Møller H, Husband K, Odell EW. Predicting malignant progression in clinically high-risk lesions by DNA ploidy analysis and dysplasia grading. Sci Rep. 2018; 8(1): 15874. https://doi. org/10.1038/s41598-018-34165-5

5. Cowan C, Gregg T, Napier S, McKenna S, Kee F. Potentially malignant oral lesions in Northern Ireland: a 20 -year population-based perspective of malignant transformation. Oral Dis. 2001; 7(1): 18-24.

6. Tilakaratne WM, Jayasooriya PR, Jayasuriya NS, De Silva RK. Oral epithelial dysplasia: Causes, quantification, prognosis, and management challenges. Periodontol 2000. 2019; 80(1): 126-147 https://doi. org/10.1111/prd.12259

7.van der Waal I. Oral leukoplakia, the ongoing discussion on definition and terminology. Med Oral Patol Oral Cir Bucal. 2015; 20(6): e685-92. https://doi.org/10.4317/medoral.21007

8. Axéll T. Occurrence of leukoplakia and some other oral white lesions among 20,333 adult Swedish people. Community Dent Oral Epidemiol. 1987; 15(1): 46-51. https://doi.org/10.1111/j.1600-0528.1987.tb00479.x

9. Eisen D. The clinical features, malignant potential, and systemic associations of oral lichen planus: A study of 723 patients. J Am Acad Dermatol. 2002; 46(2): 207-14. https://doi.org/10.1067/mjd.2002.120452

10. Wagner G, Rose C, Sachse MM. Clinical variants of lichen planus. J Dtsch Dermatol Ges. 2013; 11(4): 309-19. https://doi.org/10.1111/ ddg. 12031

11. Shirasuna K. Oral lichen planus: malignant potential and diagnosis. Oral Sci Int. 2014; 11: 1-7. https://doi.org/10.1016/S1348-8643(13)00030-X

12. Mignogna MD1, Fortuna G, Leuci S, Adamo D, Siano M, Makary $\mathrm{C}$, et al. Frictional keratoses on the facial attached gingiva are rare clinical findings and do not belong to the category of leukoplakia. J Oral Maxillofac Surg. 2011; 69(5): 1367-74. https://doi.org/10.1016/j. joms.2010.05.087

13. Rad M, Hashemipoor MA, Mojtahedi A, Zarei MR, Chamani G, Kakoei $\mathrm{S}$, et al. Correlation between clinical and histopathologic diagnoses of oral lichen planus based on modified WHO diagnostic criteria. Oral Surg Oral Med Oral Pathol Oral Radiol Endod. 2009; 107(6): 796-800 https://doi.org/10.1016/j.tripleo.2009.02.020

14. Waldron CA, Shafer WG. Leukoplakia revisited. A clinicopathological study of 3256 oral leukoplakias. Cancer. 1975; 36(4): 1386-1392. https://doi.org/10.1002/1097-0142(197510)36:4<1386::AID CNCR2820360430>3.0.CO;2-7

15. Mutalik S, Mutalik VS, Pai KM, Naikmasur VG, Phaik KS. Oral Leukoplakia - Is Biopsy at the Initial Appointment a Must? J Clin Diagn Res. 2014; 8(8): ZC04-ZC07. http://doi.org/10.7860/ JCDR/2014/8717.4659

16. Ghosh S, Pal S, Ghatak S, Saha S, Biswas S, Srivastava P. A Clinicopathologic and Epidemiologic Study of Chronic White Lesions in the Oral Mucosa. Ear Nose Throat J. 2017; 96(8): E13-E17. https:// doi.org/10.1177/014556131709600804

17. Aghbari SMH, Abushouk AI, Attia A, Elmaraezy A, Menshawy A, Ahmed MS, et al. Malignant transformation of oral lichen planus and oral lichenoid lesions: A meta-analysis of 20095 patient data. Oral Oncol. 2017; 68: 92-102. https://doi.org/10.1016/j.oraloncology.2017.03.012

18. González-Moles MÁ, Ruiz-Ávila I, González-Ruiz L, Ayén Á, GilMontoya JA, Ramos-García P. Malignant transformation risk of oral lichen planus: A systematic review and comprehensive metaanalysis. Oral Oncol. 2019; 96: 121-130. https://doi.org/10.1016/j. oraloncology.2019.07.012

19. Bánóczy J, Csiba A. Comparative study of the clinical picture and histopathologic structure of oral leukoplakia. Cancer. 1972; 29(5): 1230-4. https://doi.org/10.1002/1097-0142(197205)29:5<1230::AIDCNCR2820290515>3.0.CO;2-3

20. Stiewe T, Haran TE. How mutations shape p53 interactions with the genome to promote tumorigenesis and drug resistance. Drug Resist Updat. 2018; 38: 27-43. https://doi.org/10.1016/j.drup.2018.05.001

21. Nagler R, Bahar G, Shpitzer T, Feinmesser R. Concomitant analysis of salivary tumor markers - a new diagnostic tool for oral cancer. Clin 
Cancer Res. 2006; 12(13): 3979-84. https://doi.org/10.1158/1078-0432. CCR-05-2412

22. Woo SB1, Lin D. Morsicatio mucosae oris - a chronic oral frictional keratosis, not a leukoplakia. J Oral Maxillofac Surg. 2009; 67(1): 140-6. https://doi.org/10.1016/j.joms.2008.08.040

23. Chi AC, Lambert III PR, Pan Y, Li R, Vo D-T, Edwards E, et al. Is alveolar ridge keratosis a true leukoplakia?: A clinicopathologic comparison of 2,153 lesions. J Am Dent Assoc. 2007; 138(5): 641-51. https://doi. org/10.14219/jada.archive.2007.0236

24. Schulman JD, Beach MM, Rivera-Hidalgo F. The prevalence of oral mucosal lesions in U.S. adults: data from the Third National Health and Nutrition Examination Survey, 1988-1994. J Am Dent Assoc 2004; 135 1279-1286. https://doi.org/10.14219/jada.archive.2004.0403
25. Lee KH, Polonowita AD. Oral white lesions: pitfalls of diagnosis. Med J Aust. 2009; 190(5): 274-7.

26. Williams PM1, Poh CF, Hovan AJ, Ng S, Rosin MP. Evaluation of a suspicious oral mucosal lesion. J Can Dent Assoc. 2008; 74(3): 275-80.

27. Brouns EREA, Baart JA, Bloemena E, Karagozoglu H, van der Waal I. The relevance of uniform reporting in oral leukoplakia: Definition, certainty factor and staging based on experience with 275 patients. Med Oral Patol Oral Cir Bucal. 2013; 18 (1): e19-26. http://dx.doi.org/ doi: 10.4317/medoral.18756

28. Reibel J. Prognosis of oral pre-malignant lesions: significance of clinical, histopathological, and molecular biological characteristics. Crit Rev Oral Biol Med. 2003; 14(1):47-62.https://doi.org/10.1177/154411130301400105 